\title{
A NOTE ON THE PROPAGATION OF TEMPERATURE DISTURBANCES
}

BY

W. A. DAY

\author{
Hertford College, Oxford, England
}

1. Introduction. The purpose of this note is to prove two theorems which add to what was said in a recent paper of mine [1] concerning the implications of the classical theory of heat conduction for the rate of propagation of temperature disturbances. My own paper was prompted by a very interesting paper of G. Fichera's [2] in which he defended the classical theory against the accusation that it produces the paradoxical conclusion that disturbances propagate with infinite speed, and in which he drew attention to an observation of J. C. Maxwell's to the effect that the sensible propagation of heat, so far from being instantaneous, is an excessively slow process, and the time taken for heat to propagate is proportional to the square of the distance. In [1] I proved certain results about heat conduction in $\mathbf{R}^{n}$ that support Maxwell's observation. This note is concerned solely with what happens when the dimension $n=1$, and the techniques it employs depend upon Cauchy's integral formula of complex analysis.

Consider the convolution integral

$$
u(x, t)=\int_{-\infty}^{\infty} K(x-y, t) f(y) d y
$$

in which $K(x, t)$ is the fundamental solution

$$
\frac{1}{2(\pi t)^{1 / 2}} \exp \left(-\frac{x^{2}}{4 t}\right)
$$

of the heat equation and $f(x)$ is continuous and has compact support. The convolution arises, of course, as the unique bounded solution of the initial-value problem

$$
\begin{aligned}
\frac{\partial^{2} u}{\partial x^{2}} & =\frac{\partial u}{\partial t} \quad(-\infty<x<\infty, t>0), \\
u(x, 0) & =f(x) \quad(-\infty<x<\infty),
\end{aligned}
$$

where $u(x, t)$ is the temperature at the place $x$ at the time $t$, and $f(x)$ is the initial temperature.

Received December 29, 1994.

1991 Mathematics Subject Classification. Primary 35K; Secondary 80. 
The key idea of [1] is to study the dependence of $u(x, t)$ upon $t$ at a fixed $x$ lying outside the support of the initial temperature, and to ask what is the maximum value of $|u(x, t)|$ and what is the value of $t$ at which the maximum value is first attained. To this end [1] introduces the function

$$
U(x)=\sup _{t>0}|u(x, t)|
$$

which is the maximum temperature disturbance at $x$, and the function $\tau(x)$, which is the first time at which the maximum temperature disturbance is attained at $x$; thus $\tau(x)$ has the property that

$$
|u(x, \tau(x))|=U(x)
$$

and the property that

$$
|u(x, t)|<U(x) \text { for } 0<t<\tau(x) .
$$

The exact determination of $U(x)$ and $\tau(x)$ is difficult but nevertheless it is possible, in certain circumstances, to determine their asymptotic behaviour in the limit as $|x| \rightarrow \infty$. Thus, the arguments of [1], when specialised to the one-dimensional case, lead to the following conclusion, in which the integral

$$
M_{0}=\int_{-\infty}^{\infty} f(x) d x
$$

is the moment of the initial temperature of order 0: suppose that $f(x)$ is continuous and has compact support and suppose, in addition, that $f(x)$ is nonnegative and does not vanish everywhere; then

$$
U(x) \sim \frac{1}{(2 \pi e)^{1 / 2}} \frac{M_{0}}{|x|} \quad \text { and } \quad \tau(x) \sim \frac{x^{2}}{2} \quad \text { as }|x| \rightarrow \infty .
$$

The second of these asymptotic relations is in line with Maxwell's observation that the time taken for heat to propagate is proportional to the square of the distance.

The main purpose of this note is to point out that, at least in the one-dimensional case, the restriction upon the sign of $f(x)$ can be dispensed with almost entirely. Indeed, it will be shown that if $f(x)$ is continuous and has compact support and if $M_{0} \neq 0$ then

$$
U(x) \sim \frac{1}{(2 \pi e)^{1 / 2}} \frac{\left|M_{0}\right|}{|x|} \quad \text { and } \quad \tau(x) \sim \frac{x^{2}}{2} \quad \text { as }|x| \rightarrow \infty
$$

In the exceptional case in which $M_{0}=0$ the asymptotic behaviour of $U(x)$ and $\tau(x)$ depends upon the order of the first non-vanishing moment of the initial temperature. We shall confine our attention here to what happens when the moment of order 1 , which is the integral

$$
M_{1}=\int_{-\infty}^{\infty} x f(x) d x
$$


does not vanish. It will be shown that if $f(x)$ is continuous and has compact support, and if $M_{0}=0$ and $M_{1} \neq 0$, then

$$
U(x) \sim \frac{1}{4 \pi^{1 / 2}}\left(\frac{6}{e}\right)^{3 / 2} \frac{\left|M_{1}\right|}{|x|^{2}} \quad \text { and } \quad \tau(x) \sim \frac{x^{2}}{6} \quad \text { as }|x| \rightarrow \infty .
$$

On comparing the asymptotic relations (1.2) with their counterparts (1.1) we see that as the order of the first non-vanishing moment increases from 0 to 1 the decay of $U(x)$ at infinity becomes more rapid while the time $\tau(x)$ becomes shorter and, indeed, an initial temperature disturbance for which $M_{0}=0$ and $M_{1} \neq 0$ propagates more rapidly, by a factor of 3 , than one for which $M_{0} \neq 0$.

2. The asymptotic behaviour of $U(x)$. We turn to proving the assertions about $U(x)$ which are contained in the statements (1.1) and (1.2). To do so we must estimate the remainder $R_{N}(x, y, t)$ in the Taylor expansion

$$
K(x-y, t)=\sum_{n=0}^{N} K_{n}(x, t) y^{n}+R_{N}(x, y, t)
$$

where $x$ and $y$ are real, $t>0$, and

$$
K_{n}(x, t)=\frac{(-1)^{n}}{n !} \frac{\partial^{n}}{\partial x^{n}} K(x, t) .
$$

(The cases $N=0$ and $N=1$ are the ones that will be needed.) First, we extend the domain of definition of the fundamental solution by setting

$$
K(z, t)=\frac{1}{2(\pi t)^{1 / 2}} \exp \left(-\frac{z^{2}}{4 t}\right)
$$

for every complex number $z$ and every $t>0$. Then, by a familiar argument,

$$
R_{N}(x, y, t)=\frac{1}{2 \pi i} \int_{C} \frac{K(x-w, t)}{w-y}\left(\frac{y}{w}\right)^{N+1} d w
$$

where $C$ is the circle $w=r \exp (i \theta)(0 \leq \theta<2 \pi)$ in the complex $w$-plane and $r>|y|$. Since $x-w=x-r \cos \theta-i r \sin \theta$ and

$$
\begin{aligned}
(x-w)^{2} & =(x-r \cos \theta)^{2}-r^{2} \sin ^{2} \theta-2 i r \sin \theta(x-r \cos \theta) \\
& =\frac{1}{2} x^{2}-r^{2}+2\left(r \cos \theta-\frac{1}{2} x\right)^{2}-2 i r \sin \theta(x-r \cos \theta)
\end{aligned}
$$

it must be that

$$
\begin{aligned}
& K(x-w, t)=\frac{1}{2(\pi t)^{1 / 2}} \exp \left(-\frac{(x-w)^{2}}{4 t}\right) \\
& \quad=\frac{1}{2(\pi t)^{1 / 2}} \exp \left(\frac{r^{2}-\frac{1}{2} x^{2}}{4 t}\right) \exp \left(-\frac{\left(r \cos \theta-\frac{1}{2} x\right)^{2}}{2 t}\right) \exp \left(\frac{i r \sin \theta(x-r \cos \theta)}{2 t}\right)
\end{aligned}
$$


and

$$
|K(x-w, t)| \leq \frac{1}{2(\pi t)^{1 / 2}} \exp \left(\frac{r^{2}-\frac{1}{2} x^{2}}{4 t}\right) .
$$

Thus, if we return to Eq. (2.2) and estimate the remainder with the aid of this last inequality we see that

$$
\left|R_{N}(x, y, t)\right| \leq \frac{1}{2(\pi t)^{1 / 2}} \exp \left(\frac{r^{2}-\frac{1}{2} x^{2}}{4 t}\right) \frac{r}{r-|y|}\left(\frac{|y|}{r}\right)^{N+1}
$$

for every $r>|y|$.

Since $f(x)$ has compact support we can choose $a>0$ sufficiently large to ensure that $f(x)=0$ whenever $|x|>a$, and, having done so, we confine our attention to circles $C$ of radius $r>a$. Next, we multiply Eq. (2.1) through by $f(y)$ and integrate with respect to $y$ over the interval $-a \leq y \leq a$ to arrive at the equation

$$
\int_{-a}^{a} K(x-y, t) f(y) d y=\sum_{n=0}^{N} K_{n}(x, t) M_{n}+\int_{-a}^{a} R_{N}(x, y, t) f(y) d y,
$$

in which

$$
M_{n}=\int_{-\infty}^{\infty} x^{n} f(x) d x
$$

is the moment of the initial temperature of order $n$. In other words, we have proved that

$$
u(x, t)=u_{N}(x, t)+\int_{-a}^{a} R_{N}(x, y, t) f(y) d y,
$$

where $u_{N}(x, t)$ denotes the partial sum

$$
\sum_{n=0}^{N} K_{n}(x, t) M_{n} .
$$

By virtue of the estimate (2.3) it must be that

$$
\begin{aligned}
& \left|\int_{-a}^{a} R_{N}(x, y, t) f(y) d y\right| \\
& \quad \leq \frac{1}{2(\pi t)^{1 / 2}} \exp \left(\frac{r^{2}-\frac{1}{2} x^{2}}{4 t}\right) \frac{1}{r^{N}} \int_{-a}^{a} \frac{|y|^{N+1}}{r-|y|}|f(y)| d y \\
& \quad \leq \frac{1}{2(\pi t)^{1 / 2}} \exp \left(\frac{r^{2}-\frac{1}{2} x^{2}}{4 t}\right) \frac{a^{N+1}}{r^{N}(r-a)} \int_{-a}^{a}|f(y)| d y
\end{aligned}
$$

whenever $r>a$.

At this stage we suppose that $|x|>2 a$ and we choose $r=\frac{1}{2}|x|(>a)$. Then it follows from Eq. (2.4) and the inequality (2.5) that

$$
\begin{aligned}
|| u(x, t)|-| u_{N}(x, t)|| \\
\quad \leq\left|u(x, t)-u_{N}(x, t)\right| \\
\quad \leq \frac{1}{2(\pi t)^{1 / 2}} \exp \left(-\frac{x^{2}}{16 t}\right) \frac{(2 a)^{N+1}}{|x|^{N}(|x|-2 a)} \int_{-a}^{a}|f(y)| d y .
\end{aligned}
$$


Since

$$
\sup _{t>0}\left[\frac{1}{t^{1 / 2}} \exp \left(-\frac{x^{2}}{16 t}\right)\right]=2\left(\frac{2}{e}\right)^{1 / 2} \frac{1}{|x|}
$$

the inequality

$$
\begin{aligned}
& || u(x, t)|-| u_{N}(x, t)|| \\
& \quad \leq\left(\frac{2}{\pi e}\right)^{1 / 2}\left(\frac{2 a}{|x|}\right)^{N+1} \frac{1}{|x|-2 a} \int_{-a}^{a}|f(y)| d y
\end{aligned}
$$

must hold for every $t>0$, the right-hand side being independent of $t$ and of order $O\left(1 /|x|^{N+2}\right)$ as $|x| \rightarrow \infty$. It follows that

$$
U(x)=U_{N}(x)+O\left(\frac{1}{|x|^{N+2}}\right)
$$

where

$$
U_{N}(x)=\sup _{t>0}\left|u_{N}(x, t)\right| .
$$

There are now two cases to consider. Suppose first that $M_{0} \neq 0$ and put $N=0$. Then

$$
u_{0}(x, t)=K_{0}(x, t) M_{0}=\frac{1}{2(\pi t)^{1 / 2}} \exp \left(-\frac{x^{2}}{4 t}\right) M_{0}
$$

and calculation reveals that

$$
U_{0}(x)=\frac{1}{(2 \pi e)^{1 / 2}} \frac{\left|M_{0}\right|}{|x|} .
$$

Thus the order relation (2.7) tells us in this instance that

$$
U(x)=\frac{1}{(2 \pi e)^{1 / 2}} \frac{\left|M_{0}\right|}{|x|}+O\left(\frac{1}{|x|^{2}}\right)
$$

and, therefore, that

$$
U(x) \sim \frac{1}{(2 \pi e)^{1 / 2}} \frac{\left|M_{0}\right|}{|x|} \text { as }|x| \rightarrow \infty .
$$

Accordingly, the first of the asymptotic relations (1.1) is correct.

On the other hand, if $M_{0}=0$ and $M_{1} \neq 0$ we put $N=1$ to find that

$$
u_{1}(x, t)=K_{1}(x, t) M_{1}=\frac{x}{4 \pi^{1 / 2} t^{3 / 2}} \exp \left(-\frac{x^{2}}{4 t}\right) M_{1} \text {. }
$$

Calculation now reveals that

$$
U_{1}(x)=\frac{1}{4 \pi^{1 / 2}}\left(\frac{6}{e}\right)^{3 / 2} \frac{\left|M_{1}\right|}{|x|^{2}} .
$$

Hence, by virtue of the order relation (2.7),

$$
U(x)=\frac{1}{4 \pi^{1 / 2}}\left(\frac{6}{e}\right)^{3 / 2} \frac{\left|M_{1}\right|}{|x|^{2}}+O\left(\frac{1}{|x|^{3}}\right) .
$$

Thus

$$
U(x) \sim \frac{1}{4 \pi^{1 / 2}}\left(\frac{6}{e}\right)^{3 / 2} \frac{\left|M_{1}\right|}{|x|^{2}}
$$

and the first of the asymptotic relations (1.2) is correct. 
3. The asymptotic behaviour of $\tau(x)$. It remains to verify our assertions about the asymptotic behaviour of $\tau(x)$. Suppose first that $M_{0} \neq 0$ and put $N=0$. In these circumstances the inequality $(2.6)$ is equivalent to an inequality

$$
|| u(x, t)|-| K_{0}(x, t)|| M_{0}|| \leq \frac{A}{|x|(|x|-2 a)},
$$

in which $A$ is a positive constant and the inequality holds whenever $|x|>2 a$ and $t>0$. On multiplying through by $(2 \pi e)^{1 / 2}|x| /\left|M_{0}\right|$ we arrive at an inequality

$$
|v(x, t)-\phi(s)| \leq \frac{B}{|x|-2 a}
$$

in which

$$
\begin{gathered}
v(x, t)=(2 \pi e)^{1 / 2} \frac{|x|}{\left|M_{0}\right|}|u(x, t)|, \\
B=(2 \pi e)^{1 / 2} \frac{A}{\left|M_{0}\right|}, \\
s=\frac{|x|}{2 t^{1 / 2}}
\end{gathered}
$$

and

$$
\phi(s)=(2 e)^{1 / 2} s \exp \left(-s^{2}\right)
$$

The function $\phi(s)(s>0)$ is positive; it increases strictly on the interval $0<s<\frac{1}{2^{1 / 2}}$ and decreases strictly on the interval $s>\frac{1}{2^{1 / 2}}$. Thus it attains its supremum at $s=\frac{1}{2^{1 / 2}}$ and nowhere else; moreover, its supremum is $\phi\left(\frac{1}{2^{1 / 2}}\right)=1$. If $\varepsilon$ is any number lying in the interval $0<\varepsilon<1$ we define the numbers $\sigma_{1}(\varepsilon), \sigma_{2}(\varepsilon), \sigma_{3}(\varepsilon), \sigma_{4}(\varepsilon)$ by requiring that

$$
\begin{array}{lll}
\phi\left(\sigma_{1}\right)=1-2 \varepsilon & \text { and } & 0<\sigma_{1}<\frac{1}{2^{1 / 2}} \\
\phi\left(\sigma_{2}\right)=1-\varepsilon & \text { and } & 0<\sigma_{2}<\frac{1}{2^{1 / 2}} \\
\phi\left(\sigma_{3}\right)=1-\varepsilon & \text { and } & \sigma_{3}>\frac{1}{2^{1 / 2}} \\
\phi\left(\sigma_{4}\right)=1-2 \varepsilon & \text { and } & \sigma_{4}>\frac{1}{2^{1 / 2}} .
\end{array}
$$

These numbers are so ordered that $\sigma_{1}<\sigma_{2}<\sigma_{3}<\sigma_{4}$ and, in the limit as $\varepsilon \rightarrow 0$,

$$
\sigma_{1} \rightarrow \frac{1}{2^{1 / 2}}, \quad \sigma_{2} \rightarrow \frac{1}{2^{1 / 2}}, \quad \sigma_{3} \rightarrow \frac{1}{2^{1 / 2}}, \quad \sigma_{4} \rightarrow \frac{1}{2^{1 / 2}}
$$

Furthermore,

$$
\phi(s)>1-\varepsilon \text { for } \sigma_{2}<s<\sigma_{3}
$$

and

$$
\phi(s)<1-2 \varepsilon \text { for } 0<s<\sigma_{1} \text { and for } s>\sigma_{4} \text {. }
$$


Now let us confine our attention to values of $x$ for which $|x|>2 a+3 B$ and let us put $\varepsilon=3 B /(|x|-2 a)$ so that $0<\varepsilon<1$. It follows from the inequalities (3.1) and (3.4) that if $\sigma_{2}<s<\sigma_{3}$, that is, if

$$
\frac{x^{2}}{4 \sigma_{3}^{2}}<t<\frac{x^{2}}{4 \sigma_{2}^{2}}
$$

then

$$
v(x, t)>1-\frac{4 B}{|x|-2 a} .
$$

Likewise, it follows from the inequalities (3.1) and (3.5) that if $0<s<\sigma_{1}$ or $s>\sigma_{4}$, that is, if

$$
0<t<\frac{x^{2}}{4 \sigma_{4}^{2}} \quad \text { or } \quad t>\frac{x^{2}}{4 \sigma_{1}^{2}}
$$

then

$$
v(x, t)<1-\frac{5 B}{|x|-2 a} .
$$

Hence, all the values of $t$ at which the function $t \mapsto v(x, t)$ attains its supremum must lie in the interval

$$
\frac{x^{2}}{4 \sigma_{4}^{2}}<t<\frac{x^{2}}{4 \sigma_{1}^{2}} .
$$

Since, as a glance at Eq. (3.2) reveals, these are precisely the values of $t$ at which the function $t \mapsto|u(x, t)|$ attains its supremum, it must be that

$$
\frac{x^{2}}{4 \sigma_{4}^{2}}<\tau(x)<\frac{x^{2}}{4 \sigma_{1}^{2}} .
$$

On taking the limit as $|x| \rightarrow \infty$ we have $\varepsilon \rightarrow 0$ and it follows from Eq. (3.3) that

$$
\tau(x) \sim \frac{x^{2}}{2}
$$

which is the second of the relations (1.1).

Lastly, suppose that $M_{0}=0$ and $M_{1} \neq 0$ and put $N=1$. In these circumstances the inequality (2.6) is equivalent to an inequality

$$
|| u(x, t)|-| K_{1}(x, t)|| M_{1}|| \leq \frac{A}{|x|^{2}(|x|-2 a)}
$$

in which $A$ is a positive constant and the inequality holds whenever $|x|>2 a$ and $t>0$. On multiplying throughout by the factor $4 \pi^{1 / 2}\left(\frac{e}{6}\right)^{3 / 2}|x|^{2} /\left|M_{1}\right|$ we arrive at an inequality which is a counterpart to the inequality (3.1), namely

$$
|w(x, t)-\chi(s)| \leq \frac{B}{|x|-2 a},
$$

where

$$
\begin{aligned}
w(x, t) & =4 \pi^{1 / 2}\left(\frac{e}{6}\right)^{3 / 2} \frac{|x|^{2}}{\left|M_{1}\right|}|u(x, t)|, \\
s & =\frac{|x|}{2 t^{1 / 2}}
\end{aligned}
$$


and

$$
\chi(s)=8\left(\frac{e}{6}\right)^{3 / 2} s^{3} \exp \left(-s^{2}\right)
$$

The function $\chi(s)(s>0)$ is positive; it increases strictly on the interval $0<s<\left(\frac{3}{2}\right)^{1 / 2}$ and decreases strictly on the interval $s>\left(\frac{3}{2}\right)^{1 / 2}$. Thus it attains its supremum at $s=\left(\frac{3}{2}\right)^{1 / 2}$ and nowhere else; moreover, its supremum is $\chi\left(\left(\frac{3}{2}\right)^{1 / 2}\right)=1$. We can now argue on exactly the same lines as we have already used to show that all the values at which the function $t \mapsto w(x, t)$ attains its supremum must lie in an interval

$$
\frac{x^{2}}{4 \sigma_{4}^{2}}<t<\frac{x^{2}}{4 \sigma_{1}^{2}}
$$

where $\sigma_{1} \rightarrow\left(\frac{3}{2}\right)^{1 / 2}$ and $\sigma_{4} \rightarrow\left(\frac{3}{2}\right)^{1 / 2}$ as $|x| \rightarrow \infty$. It then follows that

$$
\tau(x) \sim \frac{x^{2}}{6},
$$

which is the second of the relations (1.2).

\section{REFERENCES}

[1] W. A. Day, On rates of propagation of heat according to Fourier's theory, Quart. Appl. Math. LV, 127-138 (1997)

[2] G. Fichera, Is the Fourier theory of heat propagation paradoxical?, Rend. del Circolo Mat. di Palermo 41, 5-28 (1992) 